\title{
Review of Physician Referrals to Orthopedic Spine versus Neurosurgery
}

\author{
Ajith Malige, MD, Roger Yuh, MD, Vikas Yellapu, MD, Vince Lands, MD, Barrett Woods, MD*, \\ Gbolabo Sokunbi, MD \\ Department of Orthopedic Surgery, St. Luke's University Health Network, Bethlehem, PA, \\ ${ }^{*}$ Rothman Orthopedic Institute, Philadelphia, PA, USA
}

Background: Previous studies have reported what patients value while choosing their surgeon, but there are no studies exploring the patterns of referral to spine surgeons among primary care physicians (PCPs). This study aims to identify any trends in PCPs' referral to orthopedic surgery versus neurosurgery for spinal pathology.

Methods: In total, 450 internal medicine, family medicine, emergency medicine, neurology, and pain management physicians who practice at one of three locations (suburban community hospital, urban academic university hospital, and urban private practice) were asked to participate in the study. Consenting physicians completed our 24-question survey addressing their beliefs according to pathologies, locations of pathologies, and surgical interventions.

Results: Overall, 108 physicians (24\%) completed our survey. Fifty-seven physicians (52.8\%) felt that neurosurgeons would provide better long-term comprehensive spinal care. Overall, $66.7 \%$ of physicians would refer to neurosurgery for cervical spine radiculopathy; $52.8 \%$, to neurosurgery for thoracic spine radiculopathy; and $56.5 \%$, to orthopedics for lumbar spine radiculopathy. Most physicians would refer all spine fractures to orthopedics for treatment except cervical spine fractures (56.5\% to neurosurgeons). Most physicians would refer to neurosurgery for extradural tumors (91.7\%) and intradural tumors (96.3\%). Most would refer to orthopedic surgeons for chronic pain. Finally, physicians would refer to orthopedics for spine fusion (61.1\%) and discectomy (58.3\%) and to neurosurgery for minimally invasive surgery $(59.3 \%)$.

Conclusions: Even though both orthopedic surgeons and neurosurgeons are intensively trained to treat a similar breath of spinal pathology, physicians vary in their referring patterns according to spinal pathology, location of pathology, and intended surgery. Education on the role of spine surgeons among PCPs is essential in ensuring unbiased referral patterns.

Keywords: Orthopedic, Neurosurgery, Spine, Primary care physician, Referral

Spinal disorders continue to affect millions of patients a year, causing pain and disability that permanently alter their daily life. First described by Hippocrates and the Greek, these conditions are found in an increasing number of patients complaining of neck and back pain each

Received July 15, 2019; Accepted August 20, 2019

Correspondence to: Ajith Malige, MD

Department of Orthopedic Surgery, St. Luke's University Health Network, 801 Ostrum st, Bethlehem, PA 18015, USA

Tel: +1-408-406-6595, Fax: +1-484-526-1593

E-mail: ajith.malige@gmail.com year, costing patients and our health care system millions of dollars in treatment a year. ${ }^{1-6)}$ Primary care, emergency medicine, and pain physicians are usually the first health care providers that see these patients and use a combination of physical therapy, pain medication, and lifestyle modification to help patients optimize their pain control and functional status. ${ }^{7,8)}$ When these measures do not bring the patient acceptable relief, these physicians refer the patient to spine surgeons for specialized treatment and possible surgical intervention.

Both orthopedic spine surgeons and neurosurgeons are trained in treating various spine pathologies. Some lit- 
Malige et al. Physician Referrals to Spine Surgery

Clinics in Orthopedic Surgery • Vol. 12, No. 1, $2020 \bullet$ www.ecios.org

erature suggests that neurosurgery residents may be more likely to be exposed to cervical disorders, intradural pathology, and microsurgery, while orthopedic residents are more likely to see thoracolumbar pathology, scoliosis, and kyphosis. ${ }^{9)}$ Currently, a majority of orthopedic surgeons complete dedicated additional fellowship training for spinal operations. The same is not true for graduating neurosurgeons who, more than half the time, do not complete additional dedicated spinal fellowship training, and this is probably due to several reasons stemming from exposure to spine surgery in residency or differences in regulation and credentialing between the specialties. ${ }^{9,10)}$ Surgeons in both specialties finish their 12-18 months' fellowship training, when required, to be proficient in treating disc herniation, laminectomies, spinal fusions, bone graft harvests, and spinal fracture management both operatively and nonoperatively, even if treatment algorithms vary between specialties. ${ }^{11-14)}$

The choice of surgeon for spinal surgery is often dictated by primary care physician (PCP) referral and in some scenarios by patient choice. Previous studies have reported what patients value when choosing their spine surgeon. ${ }^{15-18)}$ However, there are no studies exploring PCP referral patterns and their beliefs when recommending a specific spine surgeon to their patients. Our study aims to identify any trends and differences in PCPs' perception of orthopedic versus neurosurgery spine surgeons and how it affects referral patterns.

\section{METHODS}

Approval for this survey-based study was obtained both from the St. Luke's University Health Network IRB Committee and the Thomas Jefferson University School of Medicine IRB Committee. Surveys were distributed electronically at our suburban community hospital, urban academic university hospital, and urban private practice. Overall, 450 internal medicine, family medicine, emergency medicine, neurology, and pain management physicians who practice at one of our three locations were asked to participate. Physicians who consented were given our 24-question survey to complete (Supplementary Material $1)$.

The first subset of questions addresses various pathologies including cervical, thoracic, lumbar, intradural, and extradural pathologies. The second subset of questions asks which specialty a physician would refer to on the basis of the type of surgery the physician believes the patient needs. The third subset of questions looks to identify general opinions each physician has about both specialties. Finally, the fourth subset of questions looks to assess patient demographics, practice settings, and general experience.

Survey answers were anonymously stored online. Physicians who participated in our study had to answer all 24 questions in order to successfully submit the survey and be included in our sample. Descriptive statistics were used to analyze our results and determine associations IBM SPSS ver. 23.0 (IBM Corp., Armonk, NY, USA). For all analyses, a $p \leq 0.05$ denotes statistical significance.

\section{RESULTS}

Overall, 108 physicians completed our survey (24\% response rate). Most of the surveys were filled out by family medicine physicians $(\mathrm{n}=46,42.6 \%)$ and internal medicine physicians ( $\mathrm{n}=38,35.2 \%)$, most of whom practice in a community hospital setting $(\mathrm{n}=68,63.0 \%)$ (Table 1$)$. Most physicians reported either having less than 5 years of experience $(\mathrm{n}=33,30.6 \%)$ or more than 20 years of experience $(\mathrm{n}=28,25.9 \%)$ and making either less than 25 (n $=42,38.9 \%)$ or $26-50(\mathrm{n}=36,33.3 \%)$ patient referrals to a spine surgeon per year (Table 2). Fifty-seven physicians $(52.8 \%)$ answered that they feel they have easy access to a comprehensive spine institute consisting of orthopedic surgeons, neurosurgeons, pain management specialists, physiatrists, and neurologists to refer their patients to. Sixty-five physicians $(60.2 \%)$ felt that orthopedic spine surgeons are easier to contact than neurosurgeons, but 57 physicians (52.8\%) felt that neurosurgeons provide better long-term comprehensive spinal care.

\begin{tabular}{|c|c|c|c|c|}
\hline \multirow{2}{*}{ Specialty } & \multicolumn{3}{|c|}{ Type of practice } & \multirow[b]{2}{*}{ Total } \\
\hline & $\begin{array}{l}\text { Academic/ } \\
\text { university }\end{array}$ & Community & Private & \\
\hline FM & $15(13.9)$ & $28(26.0)$ & $3(2.8)$ & $46(42.6)$ \\
\hline IM & $11(10.2)$ & 20 (18.5) & $7(6.5)$ & $38(35.2)$ \\
\hline EM & $3(2.8)$ & $10(9.3)$ & 0 & $13(12.0)$ \\
\hline Neurology & 0 & $4(3.7)$ & 0 & $7(6.5)$ \\
\hline PM & 0 & $6(5.6)$ & $1(0.9)$ & $4(3.7)$ \\
\hline Total & $29(26.9)$ & $68(63.0)$ & $11(10.2)$ & $108(100.0)$ \\
\hline
\end{tabular}

Values are presented as number (\%).

FM: family medicine, IM: internal medicine, EM: emergency medicine, PM: pain management. 
Malige et al. Physician Referrals to Spine Surgery

Clinics in Orthopedic Surgery • Vol. 12, No. 1, $2020 \bullet$ www.ecios.org

Table 2. Years in Practice and Number of Referrals per Year of Our Physician Sample Population

\begin{tabular}{|c|c|c|c|c|c|c|c|c|c|c|c|}
\hline \multirow{2}{*}{ Specialty } & \multicolumn{5}{|c|}{ Year in practice } & \multicolumn{5}{|c|}{ Spine referral per year } & \multirow{2}{*}{ Total } \\
\hline & $\leq 5$ & $6-10$ & $11-15$ & $16-20$ & $\geq 21$ & $\leq 25$ & $26-50$ & $51-75$ & $76-100$ & $\geq 101$ & \\
\hline $\mathrm{FM}$ & $15(13.9)$ & $7(6.5)$ & $8(7.4)$ & $3(2.8)$ & $13(12.0)$ & $20(18.5)$ & $15(13.9)$ & $7(6.5)$ & $1(0.9)$ & $3(2.8)$ & $46(42.6)$ \\
\hline IM & $13(12.0)$ & $6(5.6)$ & $6(5.6)$ & $3(2.8)$ & $10(9.3)$ & $16(14.8)$ & $13(12.0)$ & $6(5.6)$ & $2(1.9)$ & $1(0.9)$ & $38(35.2)$ \\
\hline EM & $2(1.9)$ & $3(2.8)$ & $4(3.7)$ & $2(1.9)$ & $2(1.9)$ & $3(2.8)$ & $4(3.7)$ & $3(2.8)$ & $2(1.9)$ & $1(0.9)$ & $13(12.0)$ \\
\hline Neurology & $1(0.9)$ & $3(2.8)$ & $1(0.9)$ & 0 & $2(1.9)$ & $2(1.9)$ & $4(3.7)$ & 0 & 0 & $11(0.9)$ & $7(6.5)$ \\
\hline PM & $2(1.9)$ & $1(0.9)$ & 0 & 0 & $1(0.9)$ & $1(0.9)$ & 0 & 0 & 0 & $3(2.8)$ & $4(3.7)$ \\
\hline Total & $33(30.6)$ & $20(18.5)$ & $19(17.6)$ & $8(7.4)$ & $28(25.9)$ & $42(38.9)$ & $36(33.3)$ & $16(14.8)$ & $5(4.6)$ & $9(8.3)$ & $108(100.0)$ \\
\hline
\end{tabular}

Values are presented as number (\%).

FM: family medicine, IM: internal medicine, EM: emergency medicine, PM: pain management.

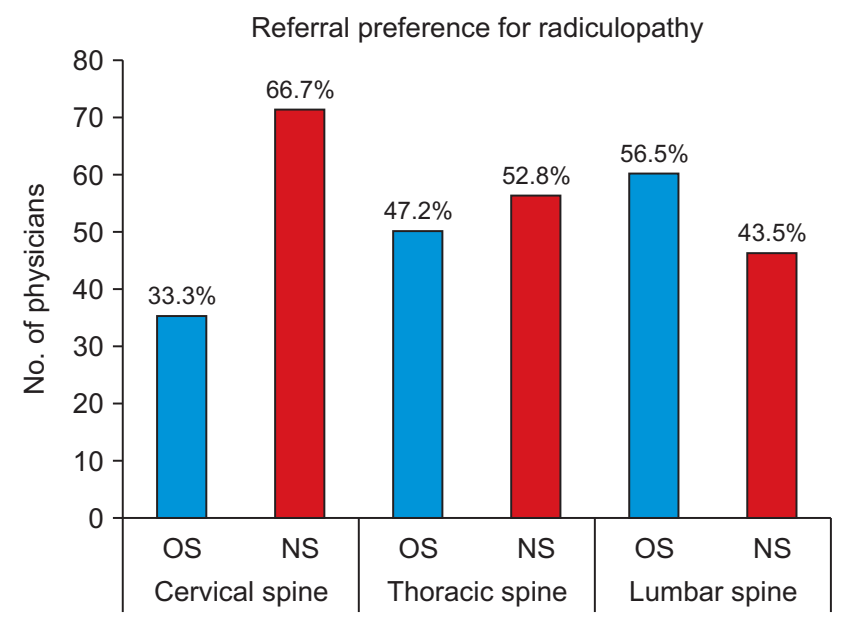

Fig. 1. Referral preference for radiculopathy in cervical spine, thoracic spine, and lumbar spine. OS: orthopaedic surgery, NS: neurosurgery.

Overall, $66.7 \%$ of physicians answered that they would refer to neurosurgery for cervical spine radiculopathy, and $52.8 \%$ of physicians would turn to neurosurgery for thoracic spine radiculopathy. However, $56.5 \%$ of physicians would refer to orthopedic surgery for lumbar spine radiculopathy (Fig. 1). A majority of physicians answered that they would refer all spine fractures (compression, thoracic, lumbar, and sacral) to an orthopedic surgeon for treatment other than cervical spine fractures, where 56.5\% of physicians would refer their patient to a neurosurgeon (Fig. 2). If a patient was found or suspected of having a spinal tumor, $91.7 \%$ of physicians would turn to neurosurgery for help in treating their patient for extradural tumors, and $96.3 \%$ of physicians would turn to neurosurgery for help in treating intradural spinal tumors.

Most PCPs answered that they would turn to orthopedic surgeons to help treat their patients with chronic

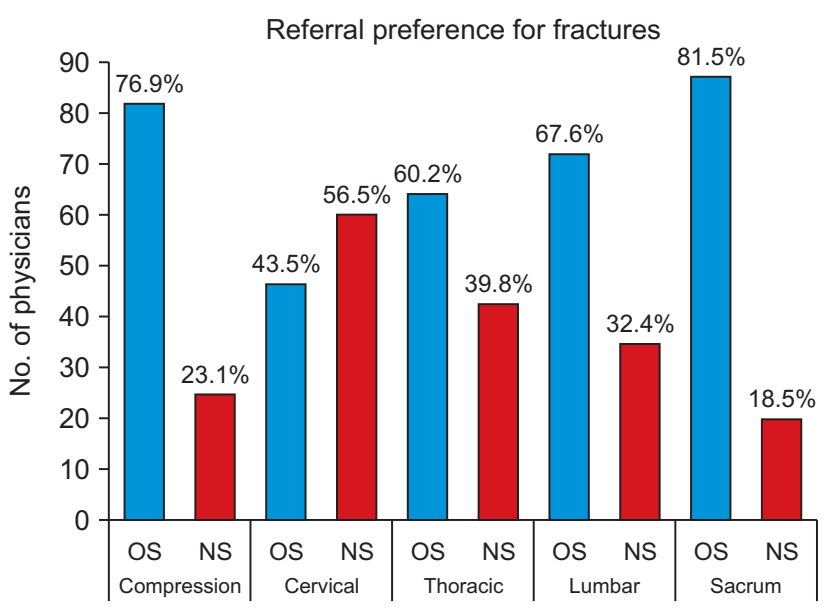

Fig. 2. Referral preference for compression fractures, traumatic cervical fractures, traumatic thoracic fractures, traumatic lumbar fractures, and traumatic sacrum fractures. OS: orthopaedic surgery, NS: neurosurgery.

pain, whether it was chronic neck and back pain (75.9\%), sacroiliac joint pain (90.7\%), or scoliosis and kyphosis structural pathology (90.7\%) (Fig. 3). Finally, if a PCP believed that a patient would eventually need a spinal fusion on the basis of clinical presentation and imaging, the physician would most likely to refer to an orthopedic spine surgeon (61.1\%). If a PCP believed a patient would need discectomy, $58.3 \%$ of physicians would refer to an orthopedic surgeon. Finally, if a PCP believed that a patient would benefit from minimally invasive surgery, 59.3\% of physicians would refer the patient to a neurosurgeon (Fig. 4).

\section{DISCUSSION}

Since the advent of internal fixation in the 1970s, coupled 
Malige et al. Physician Referrals to Spine Surgery

Clinics in Orthopedic Surgery • Vol. 12, No. 1, $2020 \bullet$ www.ecios.org

Referral preference for pain

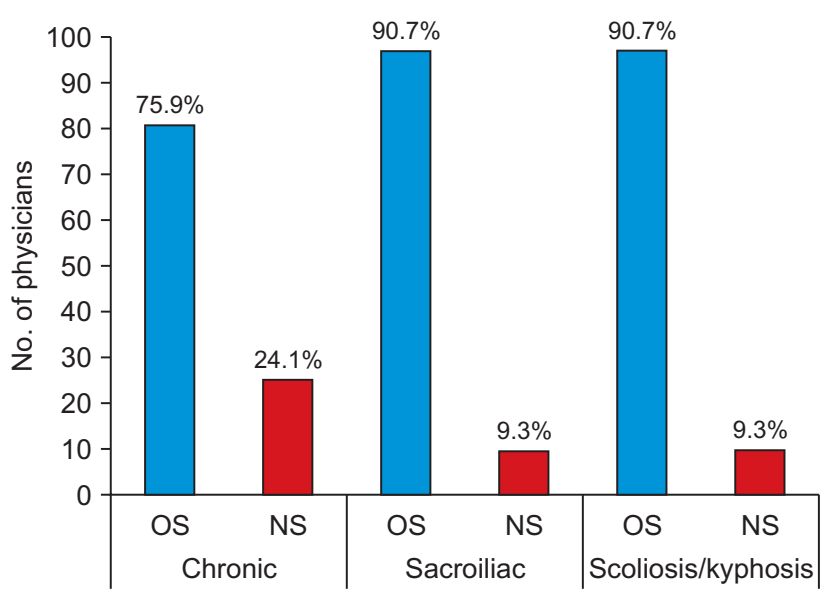

Fig. 3. Referral preference for chronic neck and back pain, sacroiliac joint pain, and scoliosis and kyphosis. OS: orthopaedic surgery, NS: neurosurgery.

with the use of anterior and posterior approaches to the spine, spine surgeons now offer more effective surgical interventions for the increasing number of patients suffering from neck and back pain. ${ }^{3,4,19)}$ The PCPs, who are now seeing and referring out many more patients to spine surgeons, are tasked with identifying ideal surgical candidates and surgeons to refer to. This survey-based study provides an analysis of PCP referral patterns on the basis of pathology, location of pathology in spine, and type of procedure that a PCP expects is needed.

Even though previous studies do show that orthopedic spine surgeons and neurosurgical spine surgeons have similar treatment and management strategies, our survey results show that overall physicians refer their patients to different specialties on the basis of the clinical situation. ${ }^{20)}$ More physicians answered that they would refer their patients with radiculopathy to neurosurgeons, except lumbar radiculopathy, possibly because they believe neurosurgeons would be better equipped to nonsurgically or surgically treat nerve root compression. However, more physicians answered that they would refer their patients to orthopedic spine surgeons for spine fractures (other than cervical spine) and chronic joint pain or structural deformity, possibly because they believe orthopedic surgeons are better equipped to nonsurgically or surgically treat bony pathology in the spine. Finally, more PCPs answered that they would refer their patients to orthopedic surgeons for spinal fusions and discectomies but not minimally invasive surgery.

While statistical tests were not done to show any statistically significant difference in referring patterns,
Referral preference by procedure

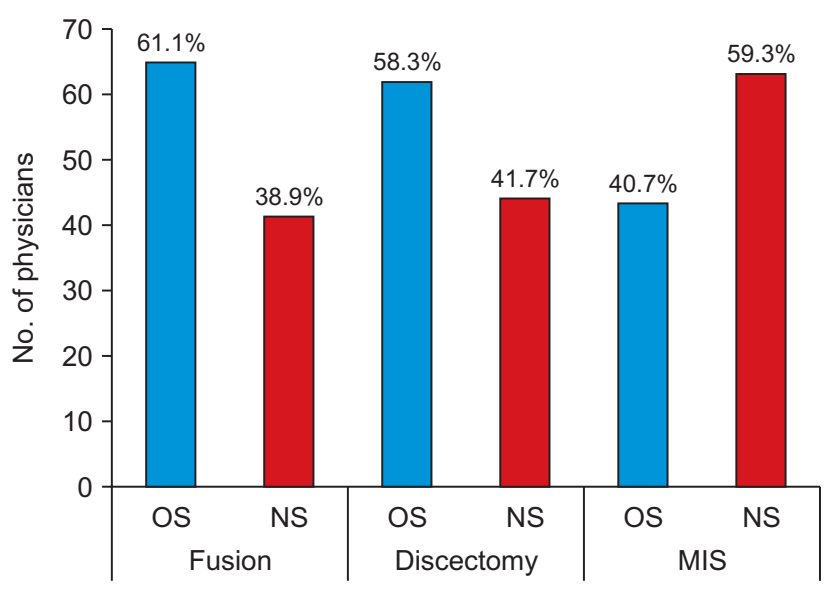

Fig. 4. Referral preference by type of procedure physician believes is necessary. OS: orthopaedic surgery, NS: neurosurgery, Fusion: spinal fusion in any spinal location, Discectomy: discectomy in any spinal location, MIS: minimally invasive surgery.

the variation by pathology, spine location, and required surgery suggests that PCPs do not believe orthopedic and neurosurgery spine surgeons are equally trained and capable of handling every spinal pathology. While residency does offer each specialty a different focus on pathology, each spine surgeon is well trained for all pathologies and operations at the completion of their training, making each surgeon capable of successfully treating each pathology (even if there are differences in comfort level among specialties). ${ }^{9-11)}$ Because of these differences in PCPs' perceptions of spine surgeons, it is important to explore what drives these perceptions and what can be done to rectify them.

PCPs' bias and referral patterns may be due in part to personal experiences with their local spine surgeons, access limitations, marketing campaigns, or hospitaldriven initiatives amongst a myriad of other factors. Various practice setting may also affect referral patterns. Better relationships with open lines of communication between spine surgeons and PCPs and education about the breath of practice and capabilities may help alleviate this discrepancy, assuring PCPs that each spine surgeon can help their patients regardless of pathology. Understanding these perceptions and pushing for unbiased referral patterns also allows for better patient access to a spine surgeon for quicker, definitive care.

Our study has a few limitations, most inherent to the survey design. Since only physicians agreeing to participate in the study were included, our cohort stands as a convenience sample. The physician response rate in our 
Malige et al. Physician Referrals to Spine Surgery

Clinics in Orthopedic Surgery • Vol. 12, No. 1, $2020 \bullet$ www.ecios.org

sample is approximately $25 \%$, a low number that is consistent with the variability of responses documented in the literature but further classifies our cohort as a convenience sample. ${ }^{21,22)}$ Finally, our survey questions were intentionally created to have binomial answers. Without having answer choices such as other or none, respondents were forced to choose between orthopedic spine surgeon and neurosurgeon, providing a distribution that might be biased.

\section{CONFLICT OF INTEREST}

No potential conflict of interest relevant to this article was reported.

\section{SUPPLEMENTARY MATERIAL}

Supplementary Material is available on the electronic version of this paper at the CiOS website, www.ecios.org.

\section{REFERENCES}

1. Marketos SG, Skiadas P. Hippocrates: the father of spine surgery. Spine (Phila Pa 1976). 1999;24(13):1381-7.

2. Tarpada SP, Morris MT, Burton DA. Spinal fusion surgery: a historical perspective. J Orthop. 2017;14(1):134-6.

3. Hoy D, March L, Brooks P, et al. The global burden of low back pain: estimates from the Global Burden of Disease 2010 study. Ann Rheum Dis. 2014;73(6):968-74.

4. Davis H. Increasing rates of cervical and lumbar spine surgery in the United States, 1979-1990. Spine (Phila Pa 1976). 1994;19(10):1117-23.

5. Agency for Healthcare Research and Quality. Healthcare cost and utilization project, HCUPnet [Internet]. Rockville, MD: Agency for Healthcare Research and Quality [cited 2019 Dec 14]. Available from: http://www.ahrq.gov/data/ hcup/.

6. Weinstein JN, Lurie JD, Olson PR, Bronner KK, Fisher ES. United States' trends and regional variations in lumbar spine surgery: 1992-2003. Spine (Phila Pa 1976). 2006; 31(23):2707-14.

7. Atlas SJ, Deyo RA. Evaluating and managing acute low back pain in the primary care setting. J Gen Intern Med. 2001;16(2):120-31.

8. Koes BW, van Tulder M, Lin CW, Macedo LG, McAuley J, Maher C. An updated overview of clinical guidelines for the management of non-specific low back pain in primary care. Eur Spine J. 2010;19(12):2075-94.

9. Dvorak MF, Collins JB, Murnaghan L, et al. Confidence in spine training among senior neurosurgical and orthopedic residents. Spine (Phila Pa 1976). 2006;31(7):831-7.

10. Daniels AH, Ames CP, Smith JS, Hart RA. Variability in spine surgery procedures performed during orthopaedic and neurological surgery residency training: an analysis of ACGME case log data. J Bone Joint Surg Am. 2014;96(23):e196.

11. Herkowitz HN, Connolly PJ, Gundry CR, Varlotta GP, Zdeblick TA, Truumees E. Resident and fellowship guidelines: educational guidelines for resident training in spinal sur- gery. Spine (Phila Pa 1976). 2000;25(20):2703-7.

12. Raja Rampersaud Y, Fisher C, Wilsey J, et al. Agreement between orthopedic surgeons and neurosurgeons regarding a new algorithm for the treatment of thoracolumbar injuries: a multicenter reliability study. J Spinal Disord Tech. 2006;19(7):477-82.

13. Grauer JN, Vaccaro AR, Beiner JM, et al. Similarities and differences in the treatment of spine trauma between surgical specialties and location of practice. Spine (Phila Pa 1976). 2004;29(6):685-96.

14. Epstein NE, Schwall G, Reillly T, Insinna T, Bahnken A, Hood DC. Surgeon choices, and the choice of surgeons, affect total hospital charges for single-level anterior cervical surgery. Spine (Phila Pa 1976). 2011;36(11):905-9.

15. Hijji FY, Narain AS, Haws BE, et al. Variation in spine surgeon selection criteria between neurosurgery and orthopedic surgery patients. Clin Spine Surg. 2018;31(2):E127-32.

16. Manning BT, Ahn J, Bohl DD, Mayo BC, Louie PK, Singh K. Spine Surgeon Selection Criteria: Factors Influencing Patient Choice. Spine (Phila Pa 1976). 2016;41(13):E814-9.

17. Harris KM. How do patients choose physicians? Evidence from a national survey of enrollees in employment-related health plans. Health Serv Res. 2003;38(2):711-32.

18. Robinson JC. The end of managed care. JAMA. 2001; 285(20):2622-8.

19. Mulholland RC, Clamp JC, Boszczyk BM. A short history of spinal training and outlook on spine speciality development in the UK 1948-2013. Eur Spine J. 2013;22 Suppl 1:S1-4.

20. Eismont FJ. The education, training, and evaluation of a spine surgeon. Spine (Phila Pa 1976). 1996;21(18):2059-63.

21. Kellerman SE, Herold J. Physician response to surveys: a review of the literature. Am J Prev Med. 2001;20(1):61-7.

22. Cummings SM, Savitz LA, Konrad TR. Reported response rates to mailed physician questionnaires. Health Serv Res. 2001;35(6):1347-55. 